\title{
7 Medizinische Evidenz in der Praxis - Was können niedergelassene Ärzte von Digital Health erwarten?
}

\author{
Tim Züwerink
}

\section{Digital Health 2017 in deutschen Arztpraxen - Anspruch und Wirklichkeit}

Immer häufiger ist von einer bevorstehenden „Revolution“ der Arztpraxis durch Digital Health die Rede. Begriffe wie „Big Data“ und künstliche Intelligenz wecken neben Datenschutzbedenken auch große Hoffnung in Bezug auf eine bessere Versorgung. Auf nicht wenige Ärzte im niedergelassenen (ndgl.) Bereich wirken sie allerdings zunächst abschreckend. Sie assoziieren damit ein Hinterfragen ihrer Kompetenz bis hin zum schrittweisen Ersatz von ihnen zugeordneten Funktionen durch digitale Werkzeuge. Auf der anderen Seite wünschen sich die Ärzte aber eine stärkere Unterstützung durch intelligente IT, von Statistiken über das Verhalten anderer Ärzte bei bestimmten Patientenprofilen, über sichere Arzneimittelinformationen, bis zur vereinfachten Kommunikation mit Patienten in einer Online-Sprechstunde (vgl. Ärzte-Zeitung 2017a).

Dies wird gespiegelt in einer steigenden Erwartungshaltung der Patienten, die sich im Internet über Gesundheitsthemen informieren, nach einfachen Möglichkeiten der Kommunikation mit ihren Ärzten suchen und auch - vorbei an der Regel-Versorgung - Digital Health Apps unterschiedlichster Anbieter und Anwendungszwecke nutzen (vgl. Ärzte-Zeitung 2017b).

Klar ist: die stärkere IT-Unterstützung nicht nur von Verwaltung und Dokumentation (wie heute schon weitgehend gegeben), sondern auch von medizinischen Entscheidungsprozessen ist eine Existenzfrage für das Gesundheitswesen. Zum einen werden die steigenden Kosten durch neue Behandlungsmethoden und den demografischen 
7 Medizinische Evidenz in der Praxis - Was können niedergelassene Ärzte von Digital Health erwarten?

Wandel ohne gleichzeitige Effizienzgewinne oder ansonsten unvermeidbare große Beitragssteigerungen nicht zu verkraften sein (Breyer 2015, 222). Zum anderen sprengt der Zuwachs und die zunehmende Differenzierung wissenschaftlicher Erkenntnisse - bis hin zur personalisierten Medizin der Zukunft - die Grenzen des menschlichen Gedächtnisses und jedes denkbaren Nachschlagewerks.

Die heutige Realität der Arztpraxen in Deutschland (und vielen anderen Ländern) lässt hier viele Wünsche offen. Es liegt stets nahe, nach der Schaffung stärkerer Anreize durch die Politik zu fragen, damit Innovationen auch in den praktischen Alltag gelangen. Dies ist notwendig und - wie erste Erfahrungen dazu aus anderen Ländern zeigen - auch möglich (zur Performance der Associated Care Organizations in den USA vgl. CMS 2016; zur Wirkung von Kopfbeitragsmodellen auf die Big-Data-Nutzung in Israel vgl. Ärzte-Zeitung 2017c). Die Skepsis gegenüber dem Einsatz neuer Technologien fordert aber vor allem attraktive Angebote mit greifbaren Mehrwerten für die Ärzte gepaart mit voller Transparenz über die innere Struktur der Lösungen und der mit ihnen verbundenen Prozesse. Dieser Artikel soll wichtige Perspektiven und Rahmenbedingungen hierfür beleuchten.

\section{Wann begeistern Innovationen den niedergelassenen Arzt?}

Unter welchen Bedingungen werden ndgl. Ärzte die Digitalisierung ihres Alltags begrüßen? Wie gelingt es am besten sie auf dieser Reise mitzunehmen? Hier soll ein Versuch der Generalisierung zweier unterscheidbarer Stoßrichtungen gemacht werden, um Digital Health-Lösungen in die Breite zu bringen (s. Tab. 1).

Diese sicher etwas pauschale Darstellung erhebt keinen Anspruch auf Vollständigkeit, soll aber den Blick auf aus Arztsicht wichtige Faktoren lenken. Fortschritt wird

\section{Tab. 1 Zwei Grundmodelle der Verbreitung von Digital Health Lösungen aus Sicht des ndgl. Arztes}

\begin{tabular}{|c|c|}
\hline Top down - Systeme & Bottom up - Lösungen \\
\hline $\begin{array}{l}\text { obligatorische Verwendung von Plattformen, } \\
\text { die große Mehrwerte für die Zukunft verspre- } \\
\text { chen }\end{array}$ & $\begin{array}{l}\text { Angebot von Werkzeugen, die schon heute greif- } \\
\text { bare (wenn auch ggf. kleinere) Mehrwerte bringen }\end{array}$ \\
\hline $\begin{array}{l}\text { Software mit umfassender Funktionalität, } \\
\text { die heutige Arbeitsabläufe grundlegend ändert }\end{array}$ & $\begin{array}{l}\text { schlanke Lösungen, die punktgenau in den Alltag } \\
\text { und die bestehende Systemlandschaft integriert } \\
\text { werden }\end{array}$ \\
\hline $\begin{array}{l}\text { „Big Data“, die dem Nutzer aggregiert als } \\
\text { statistische Entscheidungsgrundlagen dienen }\end{array}$ & $\begin{array}{l}\text { fachliche gesicherte Evidenz zur Beurteilung von } \\
\text { Einzelfällen, die zum Kontext passend präsentiert } \\
\text { wird }\end{array}$ \\
\hline $\begin{array}{l}\text { zentrale Datenspeicherung und -übermittlung } \\
\text { im Hintergrund }\end{array}$ & $\begin{array}{l}\text { volle Transparenz für und Steuerung der Daten- } \\
\text { flüsse durch den Arzt }\end{array}$ \\
\hline $\begin{array}{l}\text { Sanktionen (z.B. für jeden, der die Plattformen } \\
\text { nicht verwendet) }\end{array}$ & $\begin{array}{l}\text { Spürbarkeit und Würdigung von Verbesserungen } \\
\text { in der Versorgung durch den Einsatz der Lösungen } \\
\text { (auch finanziell) }\end{array}$ \\
\hline
\end{tabular}


es durch beide Modelle geben. Es gibt jedoch in der öffentlichen Diskussion ein Übergewicht des „Top-Down“ Modells ohne ausreichend auf die eigentlichen Anwendungsfälle für den Arzt und deren Attraktivität Bezug zu nehmen. Und letztere wird aus Sicht vieler Ärzte hauptsächlich davon bestimmt, wie leicht sich die neuen Hilfsmittel in den Alltag integrieren lassen.

Für den Durchbruch von Digital Health in der Arztpraxis braucht es auch eine Vereinbarkeit mit den heute gewohnten Prozessen. Dies kann die Etablierung völlig neuer Lösungen mit besserer Ergonomie bedeuten oder aber auch die Integration ergonomischer Lösungen in die derzeitigen Systeme.

\section{Beispiele für Anwendungsfälle der nahen Zukunft}

Welche konkreten Fortschritte können die kommenden Jahre bringen? Es steht jedenfalls zu vermuten, dass wir eine Evolution - und keine Revolution - des Versorgungsalltags sehen werden. Anhand einiger ausgewählter Anwendungsfälle, soll beschrieben werden, wie diese im „Bottom up“-Ansatz nahtlos in den Alltag der ndgl. Ärzte integriert werden können. Das Arztinformationssystem (AIS) spielt hierbei eine Schlüsselrolle in der großen Mehrzahl der Praxen:

- Erkennung seltener Erkrankungen: Es gibt immer mehr seltene Erkrankungen, v.a. weil das Wissen über die eigentlichen Ursachen gewachsen ist. So wird es für den Arzt immer schwieriger alle möglichen Diagnosen „im Kopf“ "zu haben. Hier liegt ein großes Potenzial in der Analyse der im AIS geführten Patientenakten auf mögliche Erkrankungen. Der Patient profitiert, genau wie die Kostenträger und die Hersteller der passenden Präparate.

- Leitlinien und Scores: In den 8 Minuten pro Patient, die ndgl. Ärzte im Schnitt aufbringen können (vgl. Barmer GEK 2010, 2), ist wenig Zeit für aufwendige Recherche. Die automatisierte Auswahl passender Inhalte für das im AIS aufgerufene Profil verspricht einen stärkeren Eingang der einschlägigen Evidenz in die Handlungsabläufe.

- Pre-Screening für klinische Studien (v.a. Phase III): Wenig möglicherweise passende Patienten für klinische Studien werden im ndgl. Umfeld auf eine Teilnahme angesprochen. Dies ist einer der Gründe für deren häufig verspäteten Abschluss. Ndgl. Ärzte können nicht alle möglicherweise passenden und aktuellen Studien im Gedächtnis haben. Der im AIS aufgerufene Patient kann jedoch automatisch für eine Vorprüfung der Eignung für eine klinische Studie vorgeschlagen werden und mit dessen Einverständnis an das Studienzentrum überwiesen werden (für ein Beispiel s. Abb. 1).

- Benchmarking mit anderen ndgl. Ärzten: Wo steht meine Praxis im Vergleich zu den Kollegen der gleichen Fachgruppe oder Region? Durch welche Maßnahmen kann ich meine Ökonomie und den Alltag in der Praxis optimieren? Was haben Kollegen bei vergleichbarem Fallprofil unternommen? Anonymisierte und aggregierte Daten über das Profil der Praxis können auf Wunsch des Arztes mit tausenden anderen abgeglichen werden und ihm wertvolle Einblicke liefern. 
7 Medizinische Evidenz in der Praxis - Was können niedergelassene Ärzte von Digital Health erwarten?

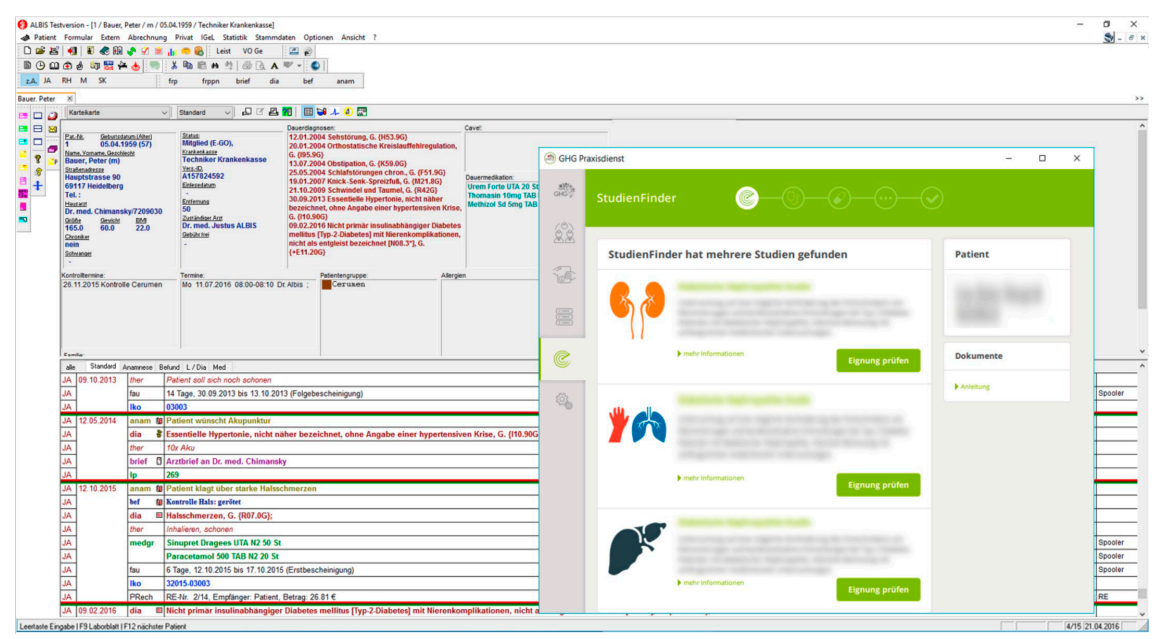

Abb. 1 Pre-Screening von Phase III-Studien im AIS „Albis“, mit angebundenem Zusatzmodul „GHG Praxisdienst" (mit freundlicher Genehmigung der CompuGroup Medical SE und Gotthardt Healthgroup AG)

\section{Eckpfeiler einer nachhaltigen Entwicklung}

Die Voraussetzungen für einen langfristigen und nachhaltigen Einsatz innovativer Technologien in der Arztpraxis orientieren sich naturgemäß an den denkbaren Risiken, hier seien nur die wichtigsten genannt:

- Einhaltung von Geist und Buchstaben der Datenschutzgesetze: Dies muss stets oberste Priorität sein im Vergleich zu allen anderen Erwägungen. Werden in (scheinbaren) Konfliktfällen Kompromisse eingegangen, erleidet das Vertrauen der Patienten und Leistungserbringer in die Lösungen erheblichen Schaden.

- Datenhoheit des Patienten: Der Patient allein und die von ihm dazu explizit ermächtigten Personen entscheiden, wer welche seiner Daten erhalten soll. Dazu brauchen die Lösungen (hier sind vor allem die elektronischen Patientenakten zu nennen) umfängliche und praktikable Rechtemanagementsysteme.

- Transparenz für den Arzt: Der Arzt muss jederzeit volle Transparenz darüber genießen, welche der von ihm generierten Informationen in welcher Form an wen kommuniziert werden. Dies muss auch retrospektiv möglich sein, d.h. der Arzt muss stets auf die volle Historie seiner Datenübermittlungen Zugriff haben. Ein Gefühl des Kontrollverlustes muss unbedingt vermieden werden.

- Finanzielle Anreize für verbesserte Versorgung: Dieser scheinbar banale Punkt bedeutet in der Endstufe eine grundlegende Umgestaltung der Vergütungssysteme weg von der reinen Leistungsvergütung hin zur Vergütungskomponenten, welche die Qualität und die Effizienz der Versorgung belohnen (zu möglichen Formen der Ausgestaltung vgl. etwa Seger u. Seger 2016).

Der Fortschritt von Digital Health setzt in der Praxis alltagstaugliche, nachhaltige Lösungen und klare Anreize und Würdigung für deren Nutzung voraus. Die praktischen Ärzte können und dürfen erwarten, dass Digital Health Lösungen sie in ihrem 
„Arztsein“ unterstützen, ihre Rolle gegenüber dem Patienten festigen und den Praxisalltag nicht nur effizienter, sondern auch angenehmer gestalten. Dies sollte in den Diskussionen über umfassende systemische Ansätze stets in Erinnerung gerufen werden.

\section{Literatur}

Ärzte-Zeitung (2017a) Digital Health. Ärzte wünschen sich Lotsen im Daten-Dickicht. URL: http://www.aerztezeitung.de/praxis_wirtschaft/e-health/article/924782/digital-health-aerzte-wuenschen-lotsen-daten-dickicht.html (abgerufen am 9.4.2017)

Ärzte-Zeitung (2017b) Digital Health. Das erwarten Patienten. URL: http://www.aerztezeitung.de/praxis_wirtschaft/internet_co/article/932692/digital-health-erwarten-patienten.html (abgerufen am 9.4.2017)

Ärzte-Zeitung (2017c) Big Data. In Israel bereits Teil der Gesundheitsversorgung. URL: http://www.aerztezeitung.de/praxis_wirtschaft/e-health/article/933027/big-data-israel-bereits-teil-gesundheitsversorgung. html (abgerufen am 12.4.2017)

Barmer GEK (2010) BARMER GEK Arztreport 2010. Pressekonferenz der BARMER GEK, URL: https://www. barmer.de/blob/38430/b696249e6e85dgdf2f714aa7842b6cd7/data/pdf-pressemappe.pdf )abgerufen am 13.4.2017)

Breyer F (2015) Demographischer Wandel und Gesundheitsausgaben: Theorie, Empirie und Politikimplikationen. In: Perspektiven der Wirtschaftspolitik 2015, 16(3), S. 215-230

CMS (2016) Medicare Accountable Care Organizations 2015 Performance Year Quality and Financial Results. Stand: 25.08.2016. URL: https://www.cms.gov/Newsroom/MediaReleaseDatabase/Fact-sheets/2016Fact-sheets-items/2016-08-25.html (abgerufen am 11.4.2017)

Seger A, Seger W (2016) Chancen, Risiken und Grenzen einer Qualitätsorientierten Vergütung im internationalen Kontext. Ein Modell für Deutschland? In: G\&S Gesundheits- und Sozialpolitik, 6/2016, S. 32-41 